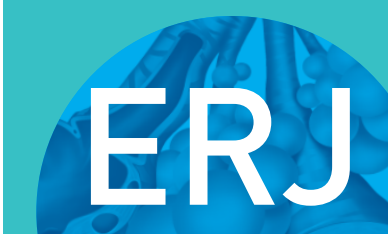

open research

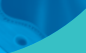

\title{
The DIAMORFOSIS (DIAgnosis and Management Of lung canceR and FibrOSIS) survey: international survey and call for consensus
}

\author{
Argyris Tzouvelekis ${ }^{1}$, Katerina Antoniou ${ }^{2}$, Michael Kreuter ${ }^{3,4}$, Matthew Evison ${ }^{5}$, \\ Torsten G. Blum $\mathbb{1}^{6}$, Venerino Poletti ${ }^{7}$, Bogdan Grigoriu $\mathbb{1}^{8}$, Carlo Vancheri ${ }^{9}$, \\ Paolo Spagnolo ${ }^{10}$, Theodoros Karampitsakos (1) ${ }^{1}$, Francesco Bonella (1) ${ }^{11}$, \\ Athol Wells ${ }^{12}$, Ganesh Raghu ${ }^{13}$, Maria Molina-Molina ${ }^{14}$, Daniel A. Culver ${ }^{15}$, \\ Elisabeth Bendstrup (10 ${ }^{16}$, Nesrin Mogulkoc ${ }^{17}$, Stefano Elia ${ }^{18}$, \\ Jacques Cadranel ${ }^{19}$ and Demosthenes Bouros $\mathbb{B}^{20}$
}

\section{ABSTRACT}

Background: Currently there is major lack of agreement on the diagnostic and therapeutic management of patients with idiopathic pulmonary fibrosis (IPF) and lung cancer. Our aim was to identify variations in diagnostic and management strategies across different institutions and provide rationale for a consensus statement on this issue.

Methods: This was a joint-survey by European Respiratory Society (ERS) Assemblies 8, 11 and 12. The survey consisted of 25 questions.

Results: Four hundred and ninety-four $(\mathrm{n}=494)$ physicians from 68 different countries and five continents responded to the survey. Ninety-four per cent of participants were pulmonologists, $1.8 \%$ thoracic surgeons and $1.9 \%$ oncologists; $97.7 \%$ were involved in multidisciplinary team approaches on diagnosis and management. Regular low-dose high-resolution computed tomography (HRCT) scan was used by $49.5 \%$ of the respondents to screen for lung cancer in IPF. Positron emission tomography (PET) scan and endobronchial ultrasound (EBUS) is performed by $60 \%$ and $88 \%$ to diagnose nodular lesions with mediastinal lymphadenopathy in patients with advanced and mild IPF, respectively. Eighty-three per cent of respondents continue anti-fibrotics following lung cancer diagnosis; safety precautions during surgical interventions including low tidal volume are applied by $67 \%$. Stereotactic radiotherapy is used to treat patients with advanced IPF (diffusing capacity of the lung for carbon monoxide $\left(D_{\mathrm{LCO}}\right)<35 \%$ ) and otherwise operable nonsmall cell lung cancer (NSCLC) by $54 \%$ of respondents and doublet platinum regimens and immunotherapy for metastatic disease by $25 \%$ and $31.9 \%$, respectively. Almost all participants (93\%) replied that a consensus statement for the management of these patients is highly warranted.

Conclusion: The diagnosis and management of IPF-lung cancer (LC) is heterogeneous with most respondents calling for a consensus statement.

@ERSpublications

The diagnosis and management of IPF-LC is heterogeneous with most survey respondents calling for a consensus statement https://bit.ly/2Smie0o

Cite this article as: Tzouvelekis A, Antoniou K, Kreuter M, et al. The DIAMORFOSIS (DIAgnosis and Management Of lung canceR and FibrOSIS) survey: international survey and call for consensus. ERJ Open Res 2021; 7: 00529-2020 [https://doi.org/10.1183/23120541.00529-2020].

This article has supplementary material available from openres.ersjournals.com.

Received: 27 July 2020 | Accepted: 23 Sept 2020

Copyright $\odot$ ERS 2021. This article is open access and distributed under the terms of the Creative Commons Attribution Non-Commercial Licence 4.0. 


\section{Introduction}

Idiopathic pulmonary fibrosis (IPF) is a debilitating fibrotic lung disease of unknown origin and pathogenesis that is steadily increasing in both incidence and mortality (40 000 patients die from IPF each year in the USA, the same as breast cancer). Until recently, there was no effective therapy for IPF, except lung transplantation [1]. Although there are two compounds licensed for the treatment of IPF that have been shown to reduce disease progression with encouraging safety and efficacy data [2, 3], neither compound has been tested prospectively in IPF patients with major comorbidities such as lung cancer [4]. Recent epidemiological evidence suggests that 3-22\% of patients with IPF develop lung cancer with an increasing risk during disease course of up to $50 \%$ and a nearly five-fold increased risk compared to the general population [5-8].This has a negative impact on patients' survival and quality of life [9-12], and most treatments for lung cancer are associated with a high morbidity and mortality in patients with IPF. Additionally, IPF and lung cancer have striking pathogenetic commonalities including microsatellite instability, epigenetic alterations, telomere attrition and impaired cellular bioenergetics [4, 13-16]. Unfortunately, there is considerable lack of agreement on the diagnostic and therapeutic management of these patients [17]. Current American Thoracic Society, European Respiratory Society, Japanese Respiratory Society and Latin American Thoracic Society (ATS/ERS/JRS/ALAT) guidelines (2018) [1] do not address this crucial issue, and there are no large randomised controlled trial data on IPF-lung cancer (LC) available [13].

We hypothesised that clinical approaches to the diagnosis and management of IPF-LC might vary substantially across different institutions internationally, with documentation of this allowing research questions to be prioritised. Thus, we conducted an international survey, called the DIAMORFOSIS (DIAgnosis and Management Of lung canceR and FibrOSIS) survey, to identify variations in diagnostic and management strategies across different institutions, raise awareness on the coexistence of these two diseases, provide rationale for a consensus statement on this issue and fuel future research and clinical study design. Some of the results have been previously published in the form of an abstract.

\section{Materials and methods}

\section{Questionnaire and participating physicians}

To identify all items to be included in the survey, we performed literature research on diagnosis, treatment and management of IPF-LC (www.ncbi.nlm.nih.gov/pubmed and www.scholar.google.com) by setting specific key words as indicated in supplementary file 1. Following this, an expert panel was formulated, encompassing respiratory physicians and oncologists from European Respiratory Society (ERS) Assemblies 8, 11 and 12 (Thoracic Surgery and Lung Transplantation, Thoracic Oncology and Interstitial Lung Diseases, respectively). Members of the expert panel were required to have experience in the diagnosis and management of IPF and lung cancer and work in reference centres of excellence for interstitial lung diseases (ILDs) and Thoracic Oncology to participate in an e-mail-based discussion to structure the survey. The final version of the questionnaire comprised 25 questions divided into five categories: 1) Participants, 2) General knowledge, 3) Diagnosis, 4) Management and 5) Future perspectives (supplementary file 2). The questionnaire was distributed through two different platforms, Google and

Affiliations: ${ }^{1}$ Dept of Internal and Respiratory Medicine, Medical School University of Patras, Patras, Greece. ${ }^{2}$ Dept of Respiratory Medicine, University of Crete, Athens, Greece. ${ }^{3}$ Center for Interstitial and Rare Lung Diseases, Pneumology, Thoraxklinik, University of Heidelberg, Heidelberg, Germany. ${ }^{4}$ German Center for Lung Research, Heidelberg, Germany. ${ }^{5}$ Manchester Thoracic Oncology Centre, Wythenshawe Hospital, Manchester University NHS Foundation Trust, Manchester, UK. 'Lungenklinik Heckeshorn, Helios Klinikum Emil von Behring, Berlin, Germany. ${ }^{7}$ Dept of Thoracic Diseases, G.B. Morgagni Hospital, Forli, Italy. ${ }^{8}$ Service des Soins Intensifs et Urgences Oncologiques \& Oncologie thoracique, Institut Jules Bordet, Centre des Tumeurs de l'Université Libre de Bruxelles, Brussels, Belgium. ${ }^{9}$ Regional Referral Centre for Rare Lung Diseases, AOU "Policlinico-Vittorio Emanuele" Dept of Clinical and Experimental Medicine, University of Catania, Catania, Italy. ${ }^{10}$ Respiratory Disease Unit, Department of Cardiac Thoracic, Vascular Sciences and Public Health, University of Padova, Padua, Italy. ${ }^{11}$ Dept of Pneumology and Allergy, Ruhrlandklinik Medical Faculty, University of Duisburg-Essen, Essen, Germany. ${ }^{12}$ Interstitial Lung Disease Unit, Dept of Respiratory Medicine, Royal Brompton and Harefield NHS Foundation Trust, London, UK. ${ }^{13}$ Center for Interstitial Lung Disease, University of Washington, Seattle, WA, USA. ${ }^{14} \mathrm{Hospital}$ Universitari de Bellvitge, L'Hospitalet de LLobregat, Barcelona, Spain. ${ }^{15}$ Cleveland Clinic, Respiratory Institute, Cleveland, $\mathrm{OH}$, USA. ${ }^{16}$ Center for Rare Lung Diseases, Dept of Respiratory Diseases and Allergy, Aarhus University Hospital, Aarhus, Denmark. ${ }^{17}$ Dept of Pulmonology, Faculty of Medicine, Ege University, İzmir, Turkey. ${ }^{18}$ Thoracic Surgery Unit, Tor Vergata University, Rome, Italy. ${ }^{19}$ Service de Pneumologie, APHP, Hôpital Tenon and Sorbonne Université, Paris, France. ${ }^{20}$ First Academic Dept of Respiratory Medicine, National and Kapodistrian University of Athens, Athens, Greece.

Correspondence: Argyris Tzouvelekis, Rion University Hospital, Rio, 26504, Greece.

E-mail: argyrios.tzouvelekisafleming.gr 
SurveyMonkey. The Google platform was used by the ERS to disseminate the questionnaire through members of the ERS Assemblies 8,11 and 12. The SurveyMonkey platform was used to disseminate the survey to other participants identified (through an internet search) to have a particular interest in ILDs and Thoracic Oncology. Results were homogenised into an Excel spreadsheet, and duplicate participants were excluded from the analysis. In both cases, participants were invited to participate through an e-mail link. The questionnaire was available from March 2019 to September 2019. Details of the questionnaire can be found in supplementary file 3 .

\section{Statistical analysis}

All questions of the survey involved categorical answers, and absolute and relative frequencies were calculated. Due to the exploratory nature of this survey and the small number of participants in country subgroups, no comparisons between participants from different countries or continents were performed, and thus no p-values were calculated. All frequencies were treated descriptively.

\section{Results}

\section{Participants}

Overall, 494 physicians from 68 different countries and five continents responded to the survey with a response rate of $28 \%$ (494 out of 1758) (figure 1). Ninety-four per cent of participants were pulmonologists, $1.8 \%$ thoracic surgeons and $1.9 \%$ thoracic oncologists; $67 \%$ and $21.6 \%$ were from University and non-University hospitals, respectively, and $51.5 \%$ were treating $>20$ patients with IPF per year. The majority of participants (63.2\%) stated a clinical experience of $>10$ years as specialists (figure 2 ).

\section{General information}

Lung cancer incidence was between 5 and 10\% of total IPF cases according to $45.3 \%$ of participants, while a big discrepancy between participants stating an incidence of $1-5 \%$ (30.8\%) and those stating an incidence $>20 \%(21.8 \%)$ was noted. Both the incidence and the type of non-lung cancer cases were unknown to a large proportion of the participants (38.8\% and $45.6 \%$, respectively). Prostate, colon and breast cancer were reported as the most common types of non-lung cancer cases in patients with IPF by $19.3 \%, 14.2 \%$ and $3.9 \%$, respectively (data not shown). The majority of the participants (54\%) declared that lower lobes are the most frequent anatomical location of the lung cancer lesions in their cohort of patients, while upper lobes were the most frequent location in $18.3 \%$. According to the majority of participants (85.2\%) nonsmall cell lung cancer (NSCLC) was the most common histological type of lung cancer in patients with IPF, with adenocarcinoma being the most frequent (58.6\%), followed by squamous (26.6\%) and small cell lung cancer (SCLC) (12.3\%) (figure 3).

\section{Diagnostic approaches}

Annual low-dose high-resolution computed tomography (HRCT) represented the most frequent screening modality for lung cancer in patients with IPF in $78.7 \%$ of participants, followed by no screening at all in
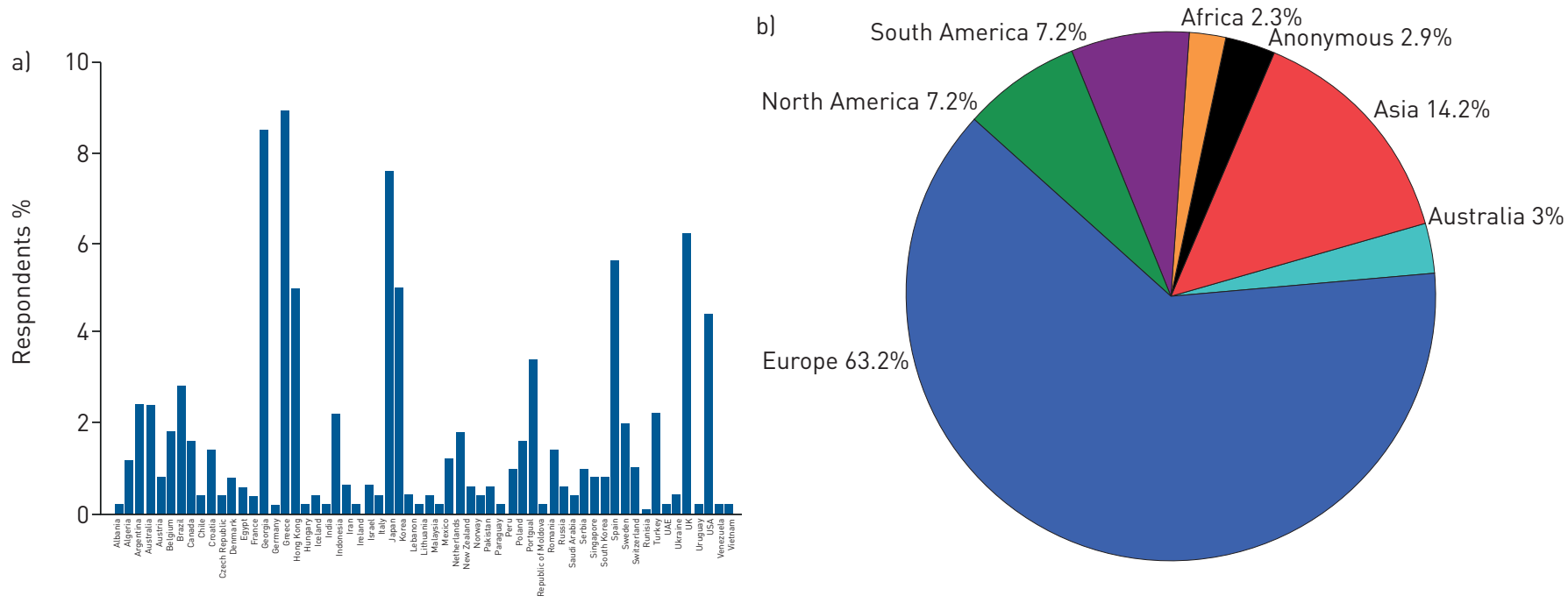

FIGURE 1 Country (a) and continent (b) of origin of participants. 
a)

What is your medical specialty?

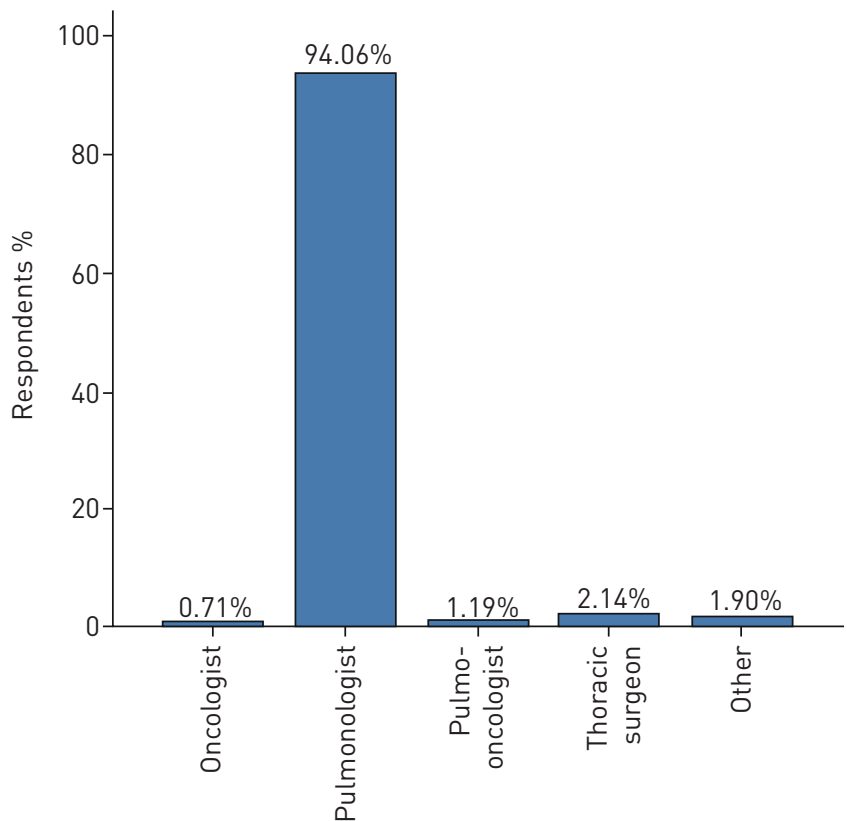

c)

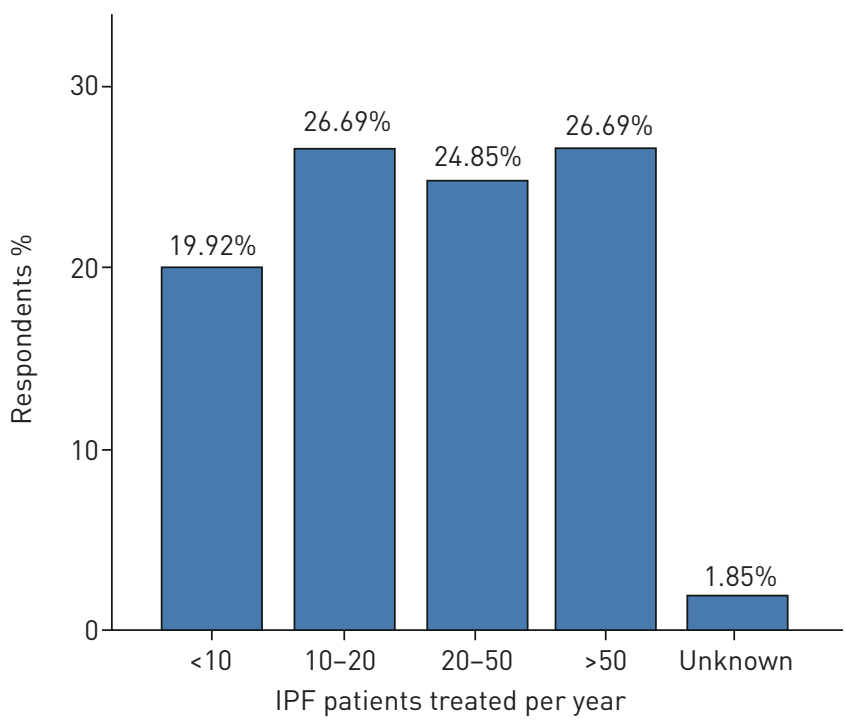

b)

What is your hospital setting?
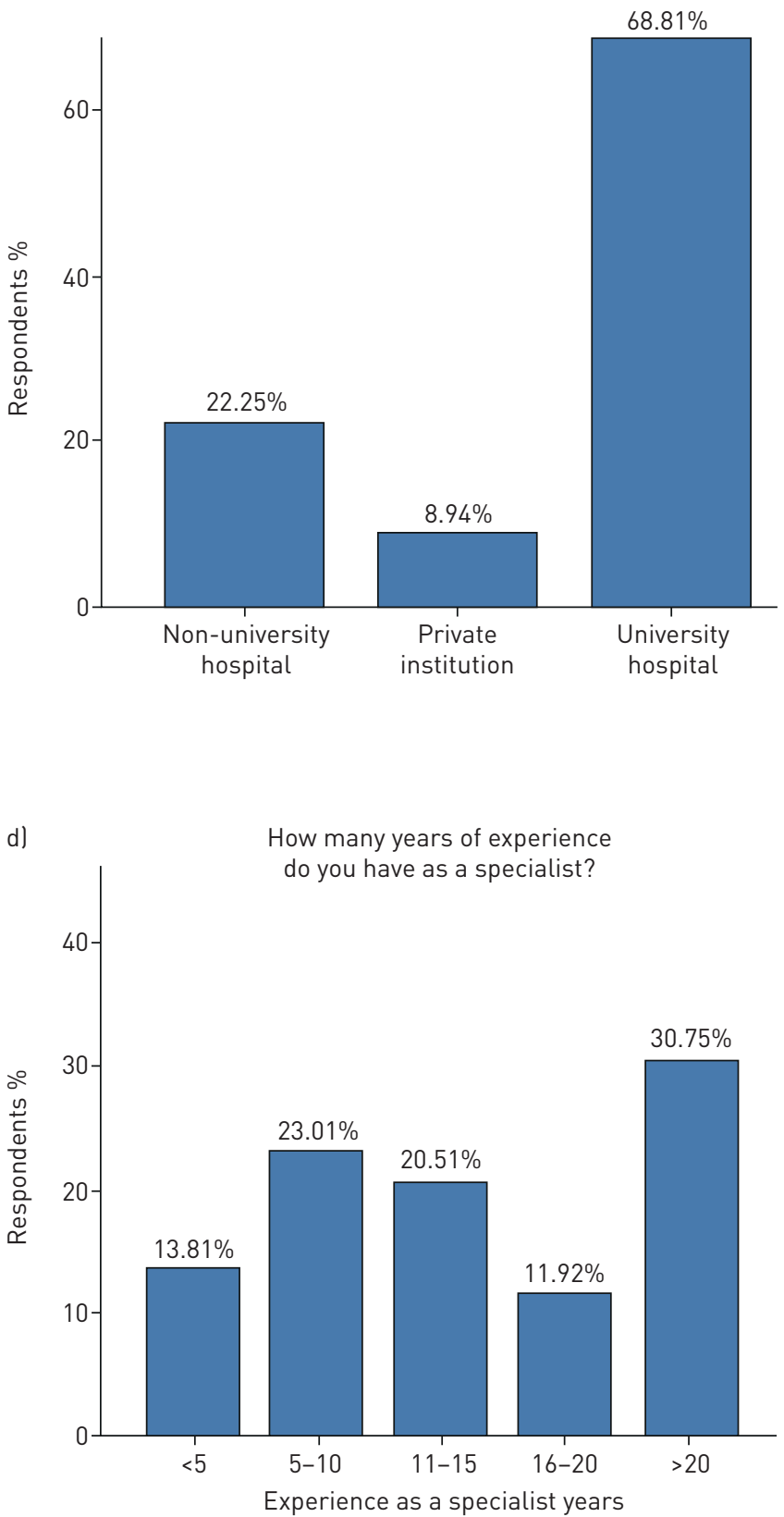

FIGURE 2 Clinical experience of participants.

$17.6 \%$ of participants. Positron emission tomography (PET) CT scan followed by endobronchial ultrasound (EBUS), in case of PET positivity, was applied as a diagnostic approach in the majority of participants in patients with $20 \mathrm{~mm}$ central nodular lesions and IPF of either mild-to-moderate (forced vital capacity $(\mathrm{FVC})>50 \%$, diffusing capacity of the lung for carbon monoxide $\left(D_{\mathrm{LCO}}\right)>35 \%$ ) or severe (FVC $<50 \%, D_{\text {LCO }}<35 \%$ ) functional impairment $(87.9 \%$ and $59.7 \%$, respectively); yet this approach was performed more often in mild-to-moderate disease than in severe disease. Median latency time between diagnosis of IPF and lung cancer was $>12$ months, as stated by $57 \%$ of respondents, while it was unknown in $27.7 \%$ (figure 4 ).

\section{Management procedures}

Multidisciplinary approaches for the management of patients with IPF-LC were applied by $78.2 \%$ of respondents. The majority of participants $(83.8 \%)$ continued treatment with anti-fibrotics when a patient 
a)

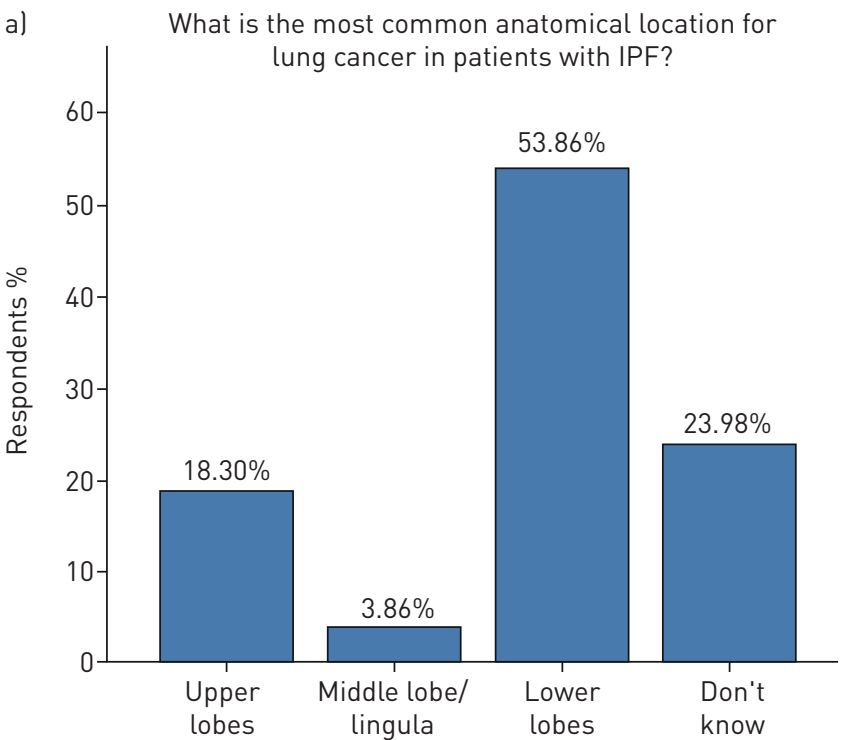

c)

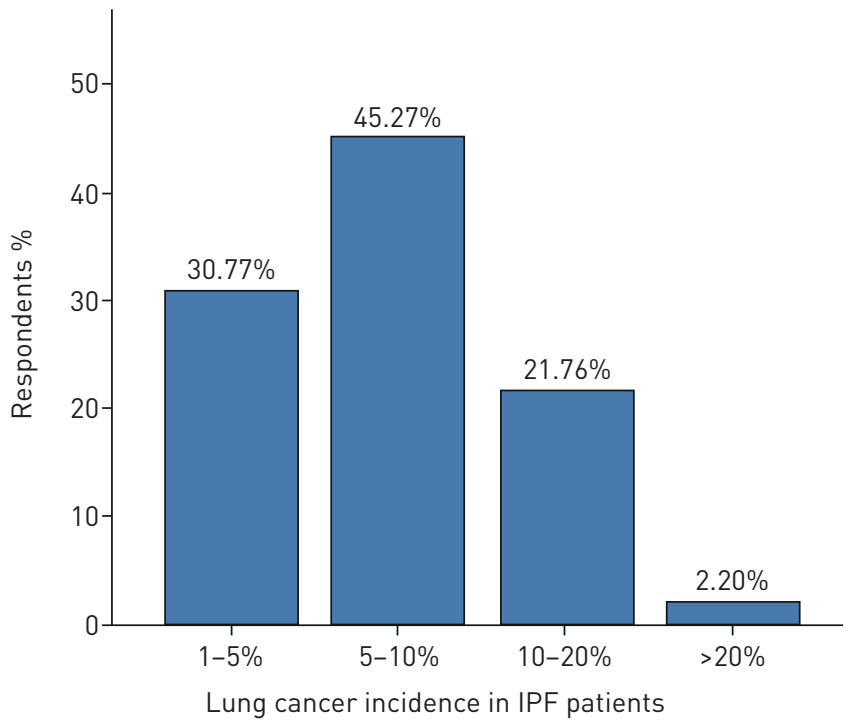

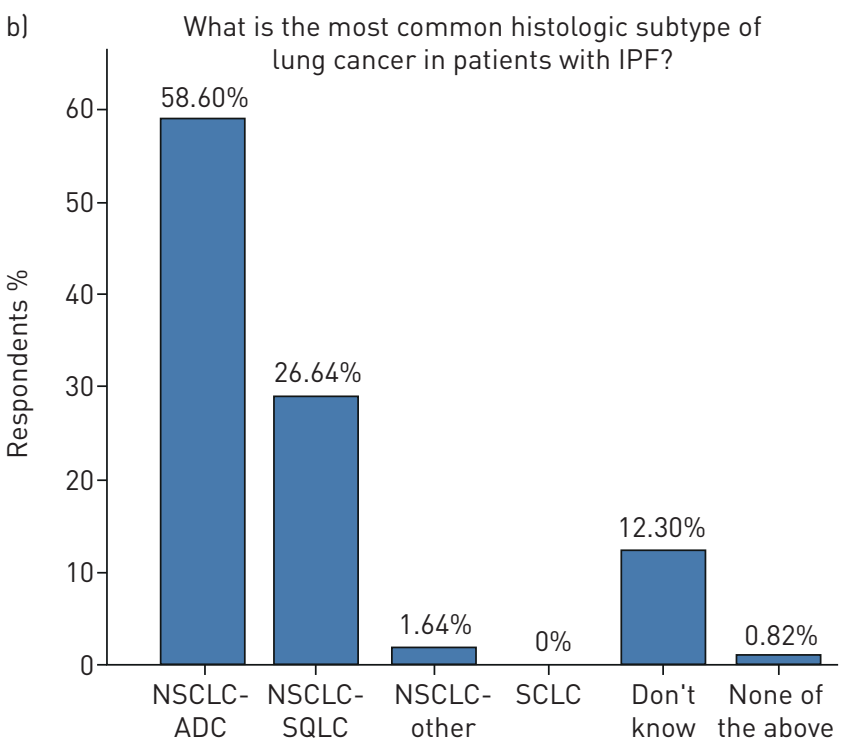

d)

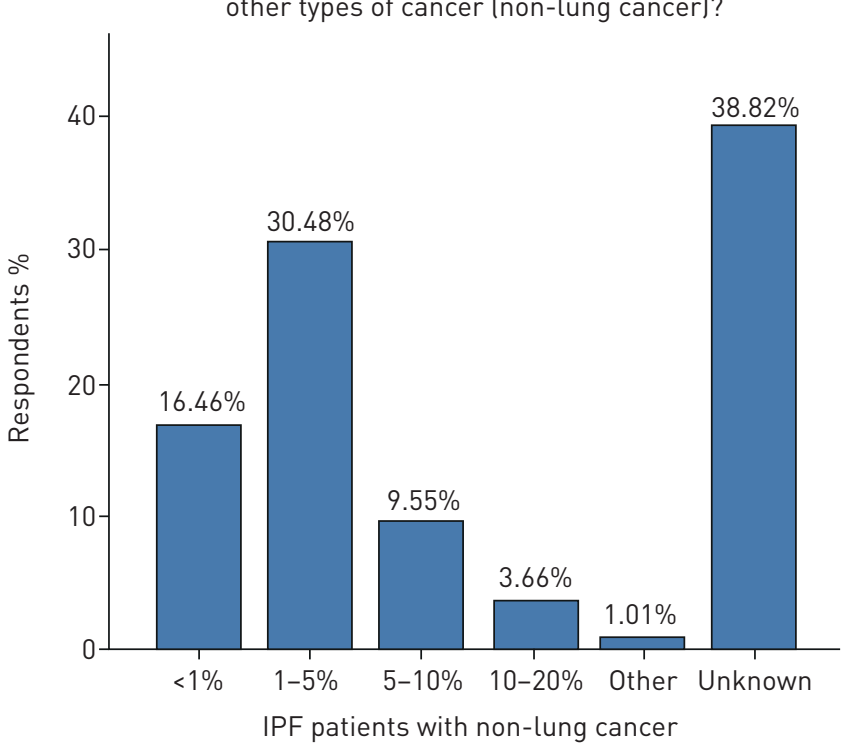

FIGURE 3 General information on patients. IPF: idiopathic pulmonary fibrosis; NSCLC: nonsmall cell lung cancer; ADC: adenocarcinoma; SQLC: squamous cell lung cancer; SCLC: small cell lung cancer.

with IPF was diagnosed with lung cancer. Major disagreement was noted in whether moderate or severe IPF is an absolute contraindication for radiotherapy or chemotherapy in patients with locally advanced NSCLC, with $40.2 \%$ of participants disagreeing, $37.2 \%$ agreeing and $20.2 \%$ stating uncertainty (figure 5). In a patient with IPF of mild-to-moderate functional impairment (FVC $\left.>50 \%, D_{\mathrm{LCO}}>35 \%\right)$ diagnosed with otherwise operable NSCLC (tumour, node, metastasis (TNM) stage I-II), surgery, stereotactic radiotherapy (depending on the cancer stage) and continuation of anti-fibrotics were the three most frequent management approaches in $78.2 \%, 40.5 \%$ and $40 \%$ of the participants, respectively. On the other hand in a patient with advanced IPF (FVC $<50 \%, D_{\text {LCO }}<35 \%$ ) and otherwise operable NSCLC (TNM stage I-II) the three most frequent management strategies were stereotactic radiotherapy, continuation of anti-fibrotics and palliative care in $54.1 \%, 37.6 \%$ and $30.6 \%$, respectively, while 1 in $5(21.4 \%)$ participants performed surgical interventions. In the case of both advanced IPF and lung cancer (TNM stage IV) the majority of participants (69\%) applied palliative care, followed by anti-fibrotics (37\%), immune checkpoint inhibitors (31.9\%) and targeted therapy (35.4\%). Doublet platinum regimens and immunotherapy for metastatic disease were chosen by $25 \%$ and $31.9 \%$, respectively (figure 6 ). 
a)

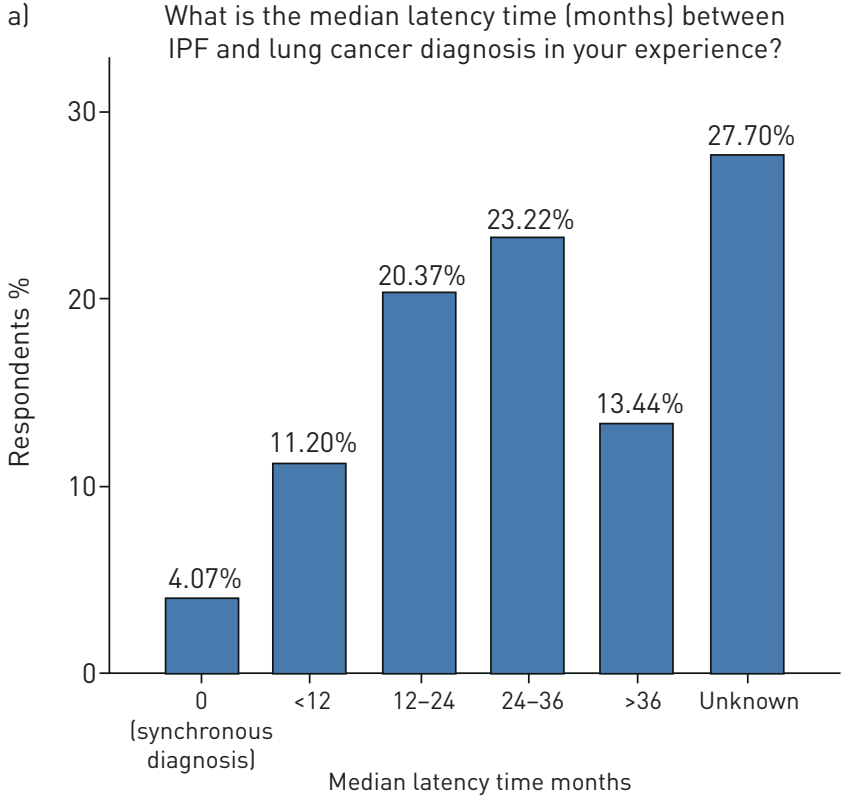

c) What would be your next diagnostic step in a patient with mild-to-moderate IPF $\left(D_{\mathrm{LCO}}>35 \%, \mathrm{FVC}>50 \%\right)$ with a central nodular lesion of $20 \mathrm{~mm}$ and mediastinal lymphadenopathy?

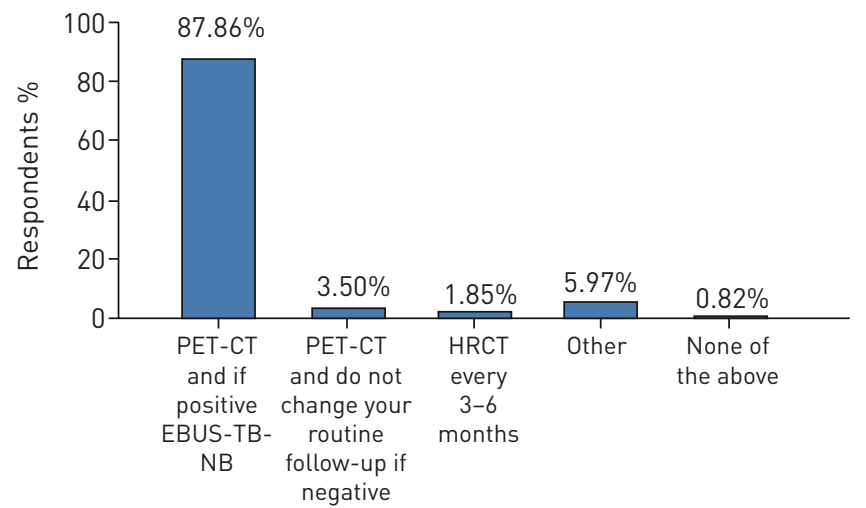

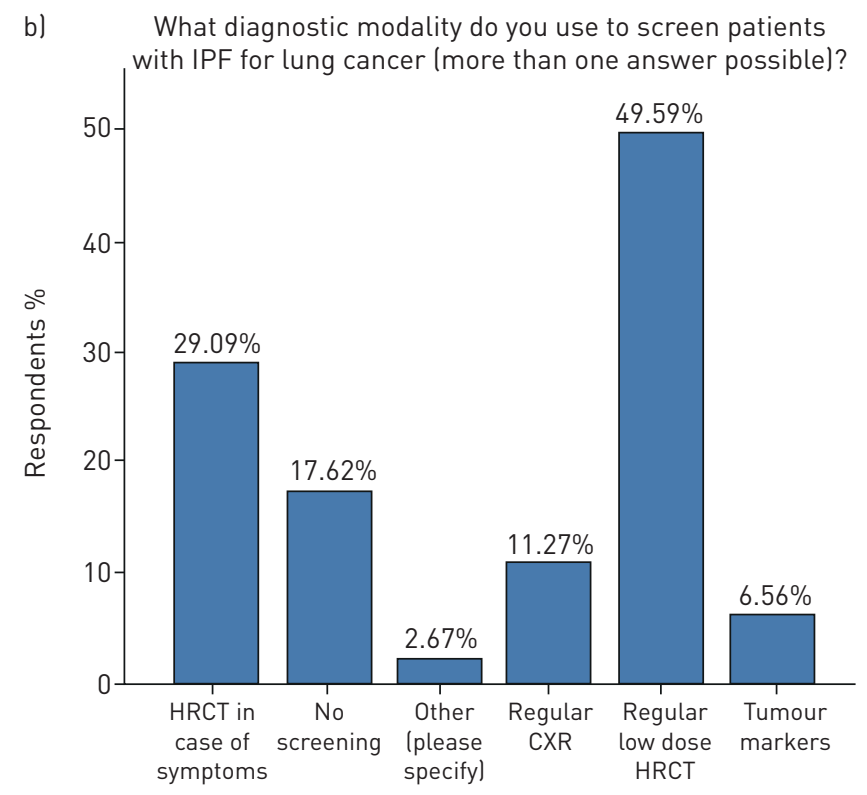

d) What would be your next diagnostic step in a patient with severe IPF $\left(D_{\mathrm{LCO}}<35 \%, \mathrm{FVC}<50 \%\right)$ with a central nodular lesion of $20 \mathrm{~mm}$ and mediastinal lymphadenopathy?

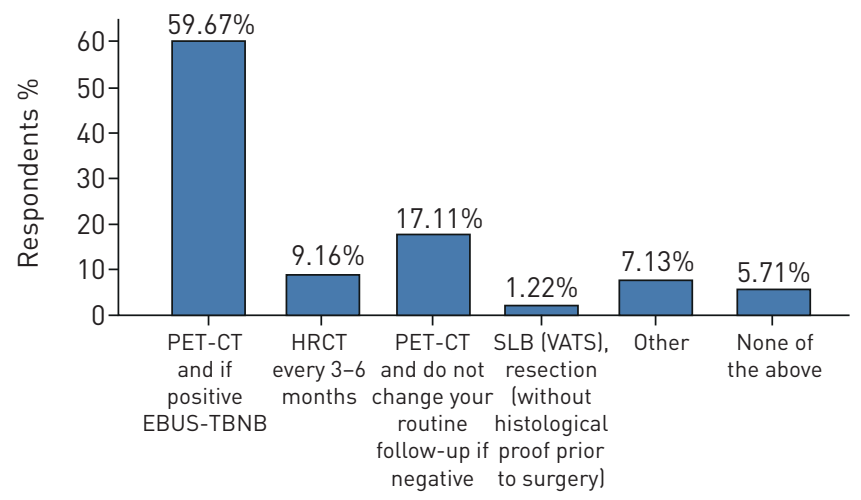

FIGURE 4 Diagnostic approaches. IPF: idiopathic pulmonary fibrosis; HRCT: high-resolution computed tomography; CXR: chest radiograph; $D_{\mathrm{LCO}}$ diffusing capacity of the lung for carbon monoxide; FVC: forced vital capacity; PET-CT: positron emission tomography/computed tomography; EBUS-TBNB: endobronchial ultrasound/transbronchial needle biopsy; SLB (VATS): surgical lung biopsy (video-assisted thoracoscopic surgery).

Regarding pre- and peri-operative safety precautions, continuation of anti-fibrotics, low tidal volume, avoidance of high fraction of inspired oxygen and minimal surgical interventions represented the most frequently applied approaches in $67.1 \%, 55.1 \%, 45.5 \%$ and $30.6 \%$ of participants, respectively (figure 5 ).

\section{Future perspectives}

Based on the vast majority of participants (92.9\%), a consensus statement for the diagnosis and management of patients with IPF-LC is mandatory for improved, homogeneous and standardised approaches. Further comments of particular interest provided by individual participants in the context of an open question were: the need for a global registry, the role of immune checkpoint inhibitors and targeted treatments, future research studies and clinical trials.

\section{Discussion}

Currently there is a major need for a consensus view of diagnostic and management strategies in patients with concomitant IPF and lung cancer. Our findings highlighted the variability in management approaches of patients with concomitant IPF and lung cancer, as participants reached consensus in only five items of the questionnaire. Our study demonstrated that there is a pressing need for increased awareness as well, given that in our survey only $28 \%$ of physicians responded to the invitation. To this 
a)

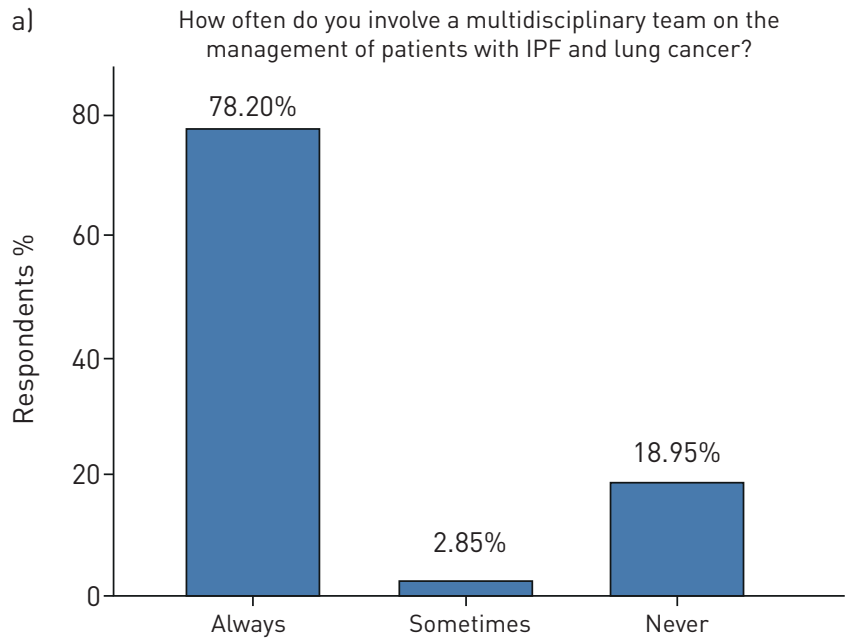

c) Do you agree with the following statement: moderate-to-severe IPF is an absolute contraindication to radiotherapy or chemotherapy in locally advanced NSCLC?

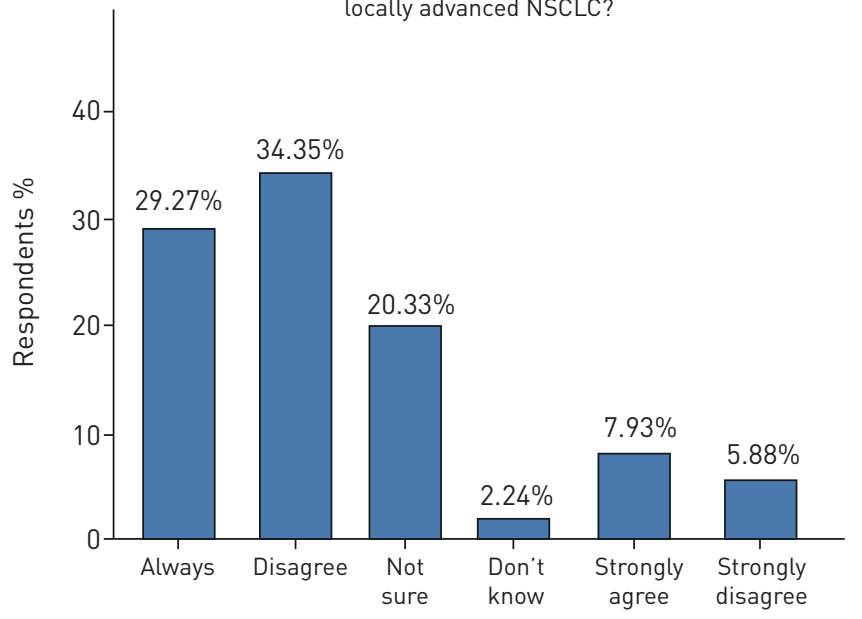

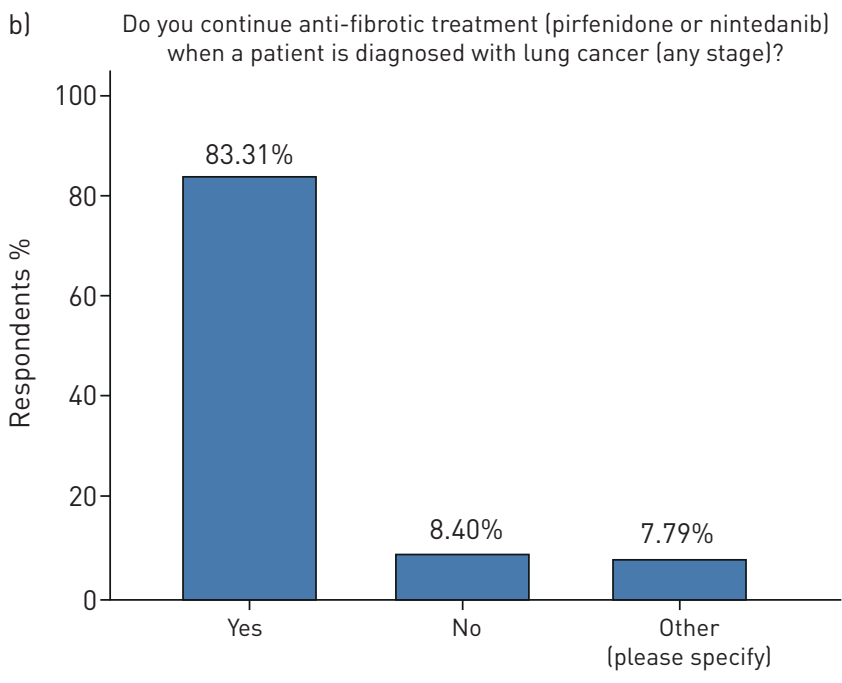

d)

Which safety precautions do you apply to patients with IPF and NSCLC undergoing surgical lung interventions?

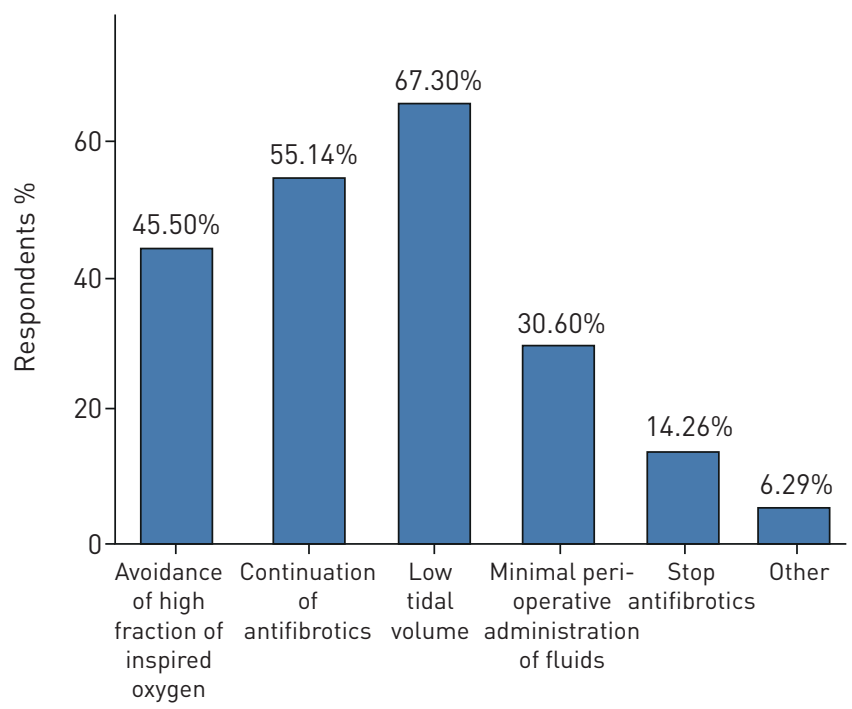

FIGURE 5 Management procedures. IPF: idiopathic pulmonary fibrosis; NSCLC: nonsmall cell lung cancer.

end, areas of uncertainty and disagreement between physicians across the world need to be identified and addressed. It is a common misconception that consensus statements need a level of data not present in this field. Our survey included a significant number of participants $(n=494)$ from six continents and identified key areas of uncertainty, as indicated by major heterogeneity in diagnostic and management practices. It also highlighted a general agreement among all participants to generate a consensus statement for harmonised approaches that will fuel clinical trials and further research.

The results of our survey could be summarised in two categories based on whether agreement between participants reached consensus, defined as per cent agreement between respondents above $75 \%$. Interestingly, our survey revealed that participants reached consensus in only five items of the questionnaire, including: 1) use of multidisciplinary discussion approaches (78.2\%); 2) continuation of anti-fibrotic treatment in patients with IPF diagnosed with lung cancer (83.8\%); 3) application of PET-CT scan and EBUS for the diagnosis of a central nodular lesion of $20 \mathrm{~mm}$ in patients with mild-to-moderate IPF and mediastinal lymphadenopathy $(87.9 \%)$; 4) surgical lung interventions in mild-to-moderate IPF cases (78.2\%); and 5) need for a consensus statement (92.9\%). This observation confirms our initial hypothesis regarding major variability in management approaches, reflecting areas of major uncertainty and highlighting the need for a consensus statement. Regarding areas of major variability and uncertainty in management strategies these could be summarised as follows: 1) screening for lung cancer using 
a)
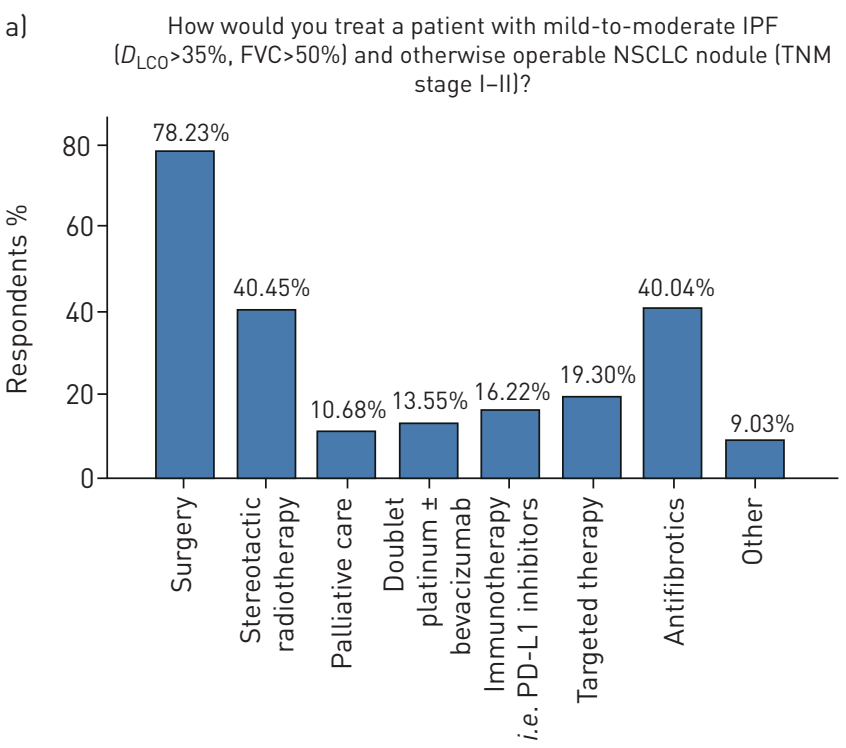

c) Do you consider any of the following treatments for advanced stage NSCLC an absolute contraindication in moderate-to-severe IPF (more than one answer possible)?

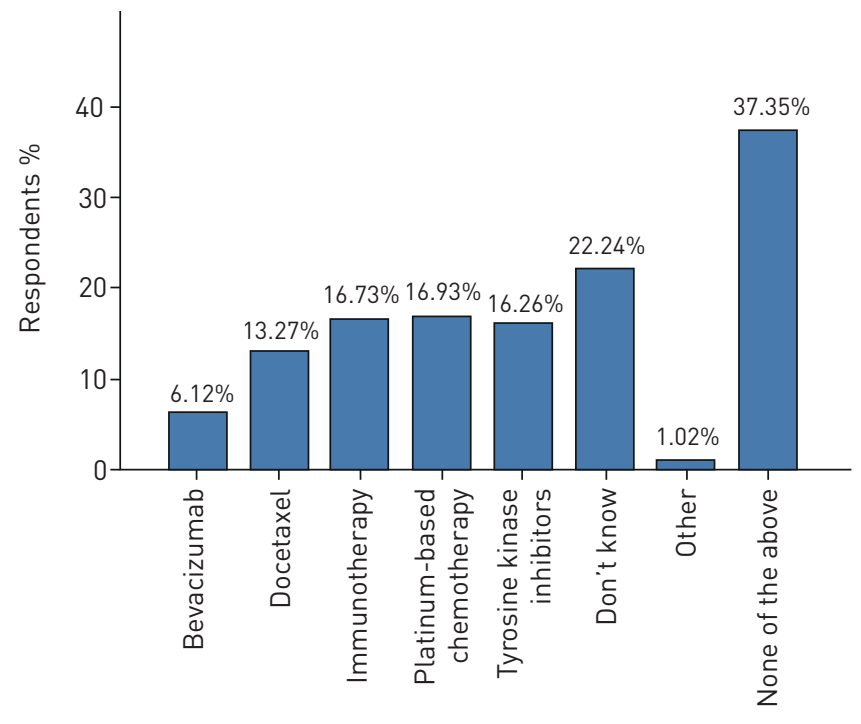

b) How would you treat a patient with mild-to-moderate IPF $\left(D_{\mathrm{LCO}}>35 \%, \mathrm{FVC}>50 \%\right)$ and otherwise operable NSCLC nodule (TNM stage I-II)?

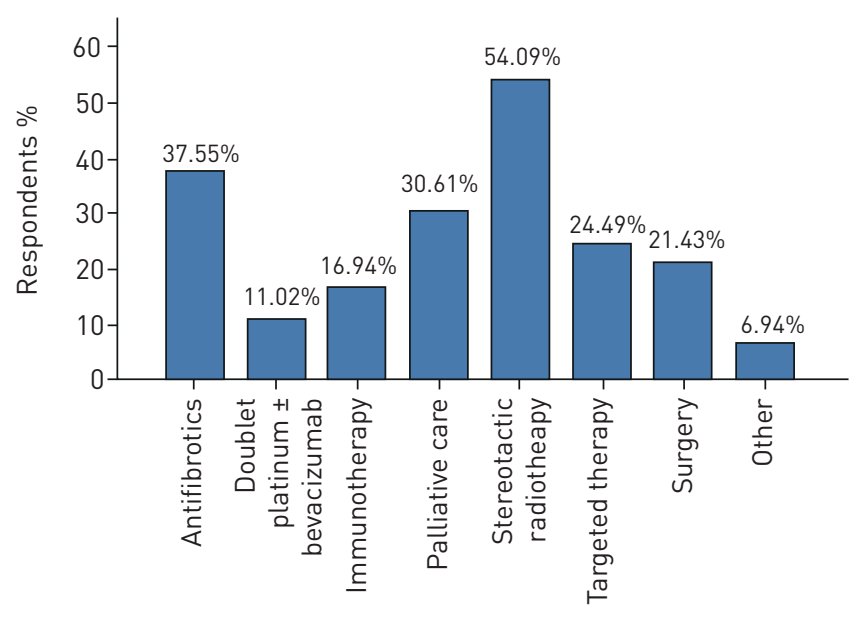

d) How would you treat a patient with advanced IPF $\left(D_{\mathrm{LCO}}>35 \%, \mathrm{FVC}<50 \%\right)$ and metastatic NSCLC (TNM stage IV)?

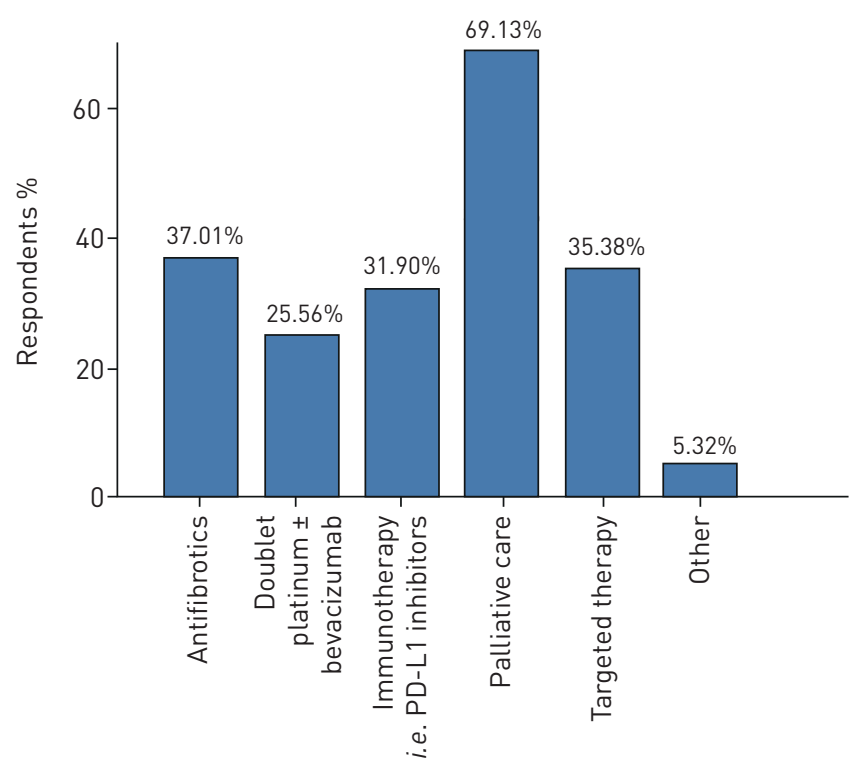

FIGURE 6 Management procedures (continued). IPF: idiopathic pulmonary fibrosis; $D_{\text {Lco: }}$ diffusing capacity of the lung for carbon monoxide; FVC: forced vital capacity; TNM: tumour, node, metastasis; NSCLC: nonsmall cell lung cancer.

low-dose HRCT on a regular (annual) basis; 2) optimal selection of patients for surgical lung interventions, chemotherapy and radiotherapy; and 3) the role of anti-fibrotics on prevention of lung cancer, treatment of lung cancer and reduction of acute exacerbations of IPF post-operatively.

Based on the latest US Preventive Services Task Force Recommendation Statement, all patients with IPF should be considered as high risk for developing lung cancer, given a much higher incidence compared to patients currently screened for lung cancer [7]. Thus, close monitoring by means of annual HRCT should be considered mandatory as it happens with other chronic lung diseases, including COPD [18]. This has become a more important issue with the implementation of novel anti-fibrotics leading to better disease outcomes and survival prolongation, given a potential accumulative incidence of lung cancer. Surprisingly, a substantial minority of participants suggested either an HRCT scan in case of additional symptoms (29.1\%) or no screening at all (17.6\%). Though it is evident in our clinical practice, as yet the benefit of screening patients with IPF by means of HRCT on a regular basis has not been proven in the context of a prospective study, as it has been shown in asymptomatic middle-aged smokers. 
Optimal selection of patients with IPF-LC for diagnostic and therapeutic interventions represents an ongoing debate. In our survey, participants agreed that patients with mild-to-moderate IPF and otherwise operable lung cancer lesions should be subjected either to bronchoscopic $(87.9 \%)$ or lung resection procedures (78.2\%) for diagnostic and therapeutic purposes, respectively, because the benefits outweigh the risks. On the contrary, agreement rates were substantially decreased in severe IPF cases with operable lung cancer lesions, where interventional procedures were suggested by $59.6 \%$ and $21.4 \%$ of respondents, for diagnosis and treatment, respectively, due to potential severe post-operative complications. Retrospective series have shown that patients with IPF exhibit higher risk for post-operative acute respiratory events than non-IPF patients [19], especially acute exacerbation [20]. Experts suggest that reduction of the duration of one-lung ventilation, videothoracoscopic surgery under spontaneous ventilation in selected patients, minimal tissue manipulation, low tidal volume ventilation strategies and avoidance of high inspiratory oxygen fraction $\left(F_{\mathrm{IO} 2}\right)$ perioperatively may exert prophylactic effects [21, 22]. In a large retrospective Japanese cohort of 1763 patients with different forms of ILDs and lung resection for lung cancer, duration and extent of surgical procedures, as well as peri-operative fraction of inspired oxygen and fluid intake, were independent risk factors of acute exacerbations [23]. To this end, a preoperative multidisciplinary evaluation should also include thoracic surgeons and anaesthesiologists in order to increase their awareness of peri- and post-operative complications of aggressive ventilation and excessive tissue manipulation, especially in patients with severely impaired lung compliance, as those with IPF. We believe that surgical lung interventions should be performed in highly selected cases based on reliable prognosticators such as functional and general performance status or composite physiological index, a multidimensional scoring system that quantifies functional and radiological impairment [14]. The deceptive nature of functional parameters in the presence of emphysema needs to be considered in this context.

Currently there are scarce data on the optimal chemotherapeutic regimen in patients with IPF-LC. In our survey only 1 in 4 participants would implement doublet platinum regimens in patients with IPF and metastatic lung cancer. Studies have shown increased pulmonary toxicity in patients with interstitial lung disease who were treated with either docetaxel or pemetrexed as well as etoposide-based regimens [24, 25]. So far, only carboplatin has shown moderate therapeutic effects with minimal toxicity [26]. A randomised controlled study (J-SONIC) investigating the efficacy of carboplatin plus nab-paclitaxel with or without nintedanib in patients with NSCLC associated with IPF is currently ongoing in Japan, and results are greatly anticipated. In the context of similar disease pathophysiology between lung cancer and IPF [15], studies investigating the effects of new immunomodulatory agents, including programmed death-ligand (PD-L) 1 inhibitors, would be of significant interest for a selective number of cases, i.e. those with upregulated PD1/PD-L1 axis [27]; yet caution should be applied considering cases of interstitial pneumonia potentially associated with nivolumab treatment $[28,29]$. Molecular testing for epidermal growth factor-receptor, KRAS and EML4-ALK mutations could also be performed for targeted treatments, as indicated by some of the participants.

With regards to radiotherapy scarce data has shown deleterious effects on patients with established lung fibrosis [9] suggesting that radiotherapy involving the lung, including stereotactic radiotherapy, should be generally avoided, unless life-threatening situations arise. Besides radiation pneumonitis, mortality after radiofrequency ablation was mainly due to pneumothoraces [30]. This is important considering that $40 \%$ of patients with IPF present with concomitant emphysema [31]. Despite these data, stereotactic radiotherapy was the predominant therapeutic intervention for severe IPF cases with operable IPF-LC lesions, as indicated by $54.1 \%$ of participants. Proton beam therapy has recently shown promising results in terms of safety in a small series of patients with IPF and lung cancer; yet the study was underpowered and retrospective [32]. Further studies are needed on the safety and efficacy of chemotherapeutic and immunomodulatory regimens as well as modern irradiation techniques including proton beam therapy.

Another challenge of the real-world clinical practice is whether anti-fibrotic agents can be combined or even synergise with chemotherapy or radiotherapy. Nintedanib has been developed as an antiangiogenic molecule and has been approved for treatment of non-squamous NSCLC [33] in combination with docetaxel-based second-line therapy. Retrospective data suggested a beneficial effect of preoperative pirfenidone on the incidence of post-operative acute exacerbations in patients with adenocarcinoma and IPF [34]. In agreement with the majority of respondents, we suggest that anti-fibrotic agents should not be discontinued during diagnostic or therapeutic workup of lung cancer, as benefits seem to outweigh the risk for unfavourable outcomes. Whether nintedanib monotherapy as cancer treatment represents a plausible strategy needs to be addressed in the context of clinical trials. Final decision should be based on multidisciplinary discussion including oncologists and on a case-by-case basis considering severity of IPF, TNM stage of lung cancer, performance status of the patient and patient's preferences.

Early implementation of palliative care may be appropriate and possibly improve patients' quality of life despite no effect on survival [35]. In agreement with current literature showing encouraging efficacy data 
in both the field of oncology [36] and lung fibrosis [37-39], the majority of participants (69.1\%) suggested palliative care as a therapeutic option, particularly in advanced cases of IPF-LC. This statement also supports the latest views that palliative care should be offered early to all patients with IPF. Larger studies using validated outcome measures are sorely needed to assess the effects of palliative care interventions on symptoms, quality of life and survival of patients with IPF-LC irrespective of disease severity.

Despite its important attributes, our survey exhibits several limitations that should be addressed cautiously. Although the study was powered by the participation of almost 500 respondents across the world, results may reflect the personal opinions of physicians and may not represent objective assessment of everyday clinical practices. Additionally, there was an over-representation of pulmonologists (94\%) while thoracic oncologists, radio-oncologists and surgeons were under-represented. Heterogeneity in answers may also mirror differences in access to treatments (targeted therapies, immune checkpoint inhibitors) and diagnostic modalities (i.e. PET-CT scan, EBUS), which may be limited in some countries due to regulatory issues. Moreover, our main aim was to survey international practices on diagnosis and treatment of patients with IPF-LC, and thus our study is unable to provide accurate epidemiological data. This needs to be addressed in the context of global registries, either retrospectively or prospectively. Such registries are more timely than ever, given that patients with IPF live longer and the incidence of lung cancer might increase. Finally, it was impossible for our survey to cover all areas of uncertainty in the field of IPF-LC. In particular, data on the impact of combined pulmonary fibrosis and emphysema (CPFE) and interstitial lung abnormalities (ILAs) on management decisions is sorely needed. Another major limitation that needs to be addressed is the lack of information on patient preferences regarding diagnostic and therapeutic interventions considering the fact that we are dealing with a very vulnerable population of patients. On the other hand, our questionnaire was anonymous and therefore answers provided are expected to be less biased.

\section{Conclusion}

In conclusion, our survey revealed major heterogeneity in diagnostic and management strategies in patients with IPF-LC, mainly arising from lack of knowledge and uncertainty in key areas of this field. ILD practitioners and oncologists almost unanimously agreed that in this poorly defined area a consensus statement for harmonised and standardised approaches is eagerly anticipated. This will fuel future trials and research studies with major impact on patients' survival and quality of life.

Acknowledgements: The authors would like to acknowledge all participants for their generous contribution and valuable feedback.

Author contributions: A. Tzouvelekis wrote the manuscript and is the main guarantor of the paper. All authors offered important intellectual contribution and approved the final form of the manuscript.

Conflict of interest: A. Tzouvelekis has received travel grants and consultation fees from Boehringer Ingelheim and Hoffmann La Roche outside the submitted work. K. Antoniou has received travel grants and consultation fees from Boehringer Ingelheim and Hoffmann La Roche. M. Kreuter has nothing to disclose. M. Evison has nothing to disclose. T.G. Blum has nothing to disclose. V. Poletti has nothing to disclose. B. Grigoriu has nothing to disclose. C. Vancheri has received unrestricted grants, and speaker and advisory board fees from Boehringer Ingelheim and F. Hoffmann-La Roche Ltd. P. Spagnolo reports grants, personal fees and nonfinancial support from Roche, PPM Services and Boehringer-Ingelheim, and reports personal fees from Red X Pharma, Galapagos and Chiesi, outside of the submitted work. T. Karampitsakos has nothing to disclose. F. Bonella has received travel grants and consultation fees from Boehringer Ingelheim, Hoffmann La Roche, Galapalagos, Savara and BMS. A. Wells has received travel grants and consultation fees from Boehringer Ingelheim and Hoffmann La Roche. G. Raghu has nothing to disclose M. Molina-Molina reports grants and payment for scientific advice for Roche, Boehringer Ingelheim, Esteve-Teijin, Chiesi, Pfizer, GSK and Galapagos. D. Culver has nothing to disclose. E. Bendstrup has nothing to disclose N. Mogulkoc has nothing to disclose. S. Elia has nothing to disclose. J. Cadranel reports grants from BI, and personal fees for consultancy and experts boards from Roche and BI, outside the submitted work. D. Bouros reports grants, personal fees, nonfinancial support and other support from BI Hellas, and other support from Roche, outside the submitted work.

\section{References}

1 Raghu G, Remy-Jardin M, Myers JL, et al. Diagnosis of Idiopathic Pulmonary Fibrosis. An official ATS/ERS/JRS/ ALAT clinical practice guideline. Am J Respir Crit Care Med 2018; 198: e44-e68.

2 Richeldi L, du Bois RM, Raghu G, et al. Efficacy and safety of nintedanib in idiopathic pulmonary fibrosis. $N$ Engl J Med 2014; 370: 2071-2082.

3 Noble PW, Albera C, Bradford WZ, et al. Pirfenidone for idiopathic pulmonary fibrosis: analysis of pooled data from three multinational phase 3 trials. Eur Respir J 2016; 47: 243-253.

4 Karampitsakos T, Tzilas V, Tringidou R, et al. Lung cancer in patients with idiopathic pulmonary fibrosis. Pulm Pharmacol Ther 2017; 45: 1-10.

5 Park J, Kim DS, Shim TS, et al. Lung cancer in patients with idiopathic pulmonary fibrosis. Eur Respir J 2001; 17: 1216-1219.

6 Le Jeune I, Gribbin J, West J, et al. The incidence of cancer in patients with idiopathic pulmonary fibrosis and sarcoidosis in the UK. Respir Med 2007; 101: 2534-2540. 
7 Ozawa Y, Suda T, Naito T, et al. Cumulative incidence of and predictive factors for lung cancer in IPF. Respirology 2009; 14: 723-728.

8 Tzouvelekis A, Karampitsakos T, Gomatou G, et al. Lung cancer in patients with idiopathic pulmonary fibrosis. A retrospective multicenter study in Greece. Pulm Pharmacol Ther 2020; 60: 101880.

9 Kreuter M, Ehlers-Tenenbaum S, Schaaf M, et al. Treatment and outcome of lung cancer in idiopathic interstitial pneumonias. Sarcoidosis Vasc Diffuse Lung Dis 2015; 31: 266-274.

10 Kreuter M, Ehlers-Tenenbaum S, Palmowski K, et al. Impact of comorbidities on mortality in patients with idiopathic pulmonary fibrosis. PLoS One 2016; 11: e0151425.

11 Kanaji N, Tadokoro A, Kita N, et al. Impact of idiopathic pulmonary fibrosis on advanced non-small cell lung cancer survival. J Cancer Res Clin Oncol 2016; 142: 1855-1865.

12 Tomassetti S, Gurioli C, Ryu JH, et al. The impact of lung cancer on survival of idiopathic pulmonary fibrosis Chest 2015; 147: 157-164.

13 Tzouvelekis A, Spagnolo P, Bonella F, et al. Patients with IPF and lung cancer: diagnosis and management. Lancet Respir Med 2018; 6: 86-88.

14 Tzilas V, Valeyre D, Tzouvelekis A, et al. Taking a giant step in the diagnosis of idiopathic pulmonary fibrosis. Lancet Respir Med 2018; 6: 82-84.

15 Vancheri C, Failla M, Crimi N, et al. Idiopathic pulmonary fibrosis: a disease with similarities and links to cancer biology. Eur Respir J 2010; 35: 496-504.

16 Vassilakis DA, Sourvinos G, Spandidos DA, et al. Frequent genetic alterations at the microsatellite level in cytologic sputum samples of patients with idiopathic pulmonary fibrosis. Am J Respir Crit Care Med 2000; 162 $1115-1119$.

17 Naccache J-M, Gibiot Q, Monnet I, et al. Lung cancer and interstitial lung disease: a literature review. J Thorac Dis 2018; 10: 3829-3844.

18 Moyer VA, U.S.P.S.T. Force. Screening for lung cancer: U.S. Preventive Services Task Force recommendation statement. Ann Intern Med 2014; 160: 330-338.

19 Sakata R, Fujii Y, Kuwano H. Thoracic and cardiovascular surgery in Japan during 2008: annual report by The Japanese Association for Thoracic Surgery. Gen Thorac Cardiovasc Surg 2010; 58: 356-383.

20 Kreuter M, Polke M, Walsh S, et al. A global perspective on acute exacerbation of idiopathic pulmonary fibrosis (AE-IPF): results from an international survey. Eur Respir J 2018; 52: Suppl. 62, OA542.

21 Choi SM, Lee J, Park YS, et al. Postoperative pulmonary complications after surgery in patients with interstitial lung disease. Respiration 2014; 87: 287-293.

22 Acute Respiratory Distress Syndrome Network, Brower RG, Matthay MA, et al. Ventilation with lower tidal volumes as compared with traditional tidal volumes for acute lung injury and the acute respiratory distress syndrome. N Engl J Med 2000; 342: 1301-1308.

23 Sato T, Teramukai S, Kondo $\mathrm{H}$, et al. Impact and predictors of acute exacerbation of interstitial lung diseases after pulmonary resection for lung cancer. J Thorac Cardiovasc Surg 2014; 147: 1604-1611 e3.

24 Watanabe N, Niho S, Kirita K, et al. Second-line docetaxel for patients with platinum-refractory advanced non-small cell lung cancer and interstitial pneumonia. Cancer Chemother Pharmacol 2015; 76: 69-74.

25 Minegishi Y, Kuribayashi H, Kitamura K, et al. The feasibility study of Carboplatin plus Etoposide for advanced small cell lung cancer with idiopathic interstitial pneumonias. J Thorac Oncol 2011; 6: 801-807.

26 Watanabe N, Taniguchi H, Kondoh Y, et al. Efficacy of chemotherapy for advanced non-small cell lung cancer with idiopathic pulmonary fibrosis. Respiration 2013; 85: 326-331.

27 Celada LJ, Kropski JA, Herazo-Maya JD, et al. PD-1 up-regulation on CD4(+) T cells promotes pulmonary fibrosis through STAT3-mediated IL-17A and TGF-betal production. Sci Transl Med 2018; 10: eaar8356.

28 Kanai O, Nakatani K, Fujita K, et al. Concurrence of nivolumab-induced interstitial lung disease and cancer invasion. Respirol Case Rep 2017; 5: e00257.

29 Kato T, Masuda N, Nakanishi Y, et al. Nivolumab-induced interstitial lung disease analysis of two phase II studies patients with recurrent or advanced non-small-cell lung cancer. Lung Cancer 2017; 104: 111-118.

30 Chen H, Senan S, Nossent EJ, et al. Treatment-related toxicity in patients with early-stage non-small cell lung cancer and coexisting interstitial lung disease: a systematic review. Int J Radiat Oncol Biol Phys 2017; 98: 622-631.

31 Cottin V, Nunes H, Brillet PY, et al. Combined pulmonary fibrosis and emphysema: a distinct underrecognised entity. Eur Respir J 2005; 26: 586-593.

32 Ono T, Hareyama M, Nakamura T, et al. The clinical results of proton beam therapy in patients with idiopathic pulmonary fibrosis: a single center experience. Radiat Oncol 2016; 11: 56.

33 Reck M, Kaiser R, Mellemgaard A, et al. Docetaxel plus nintedanib versus docetaxel plus placebo in patients with previously treated non-small-cell lung cancer (LUME-Lung 1): a phase 3, double-blind, randomised controlled trial. Lancet Oncol 2014; 15: 143-155.

34 Iwata T, Yoshida S, Fujiwara T, et al. Effect of perioperative pirfenidone treatment in lung cancer patients with idiopathic pulmonary fibrosis. Ann Thorac Surg 2016; 102: 1905-1910.

35 Kreuter M, Bendstrup E, Russell AM, et al. Palliative care in interstitial lung disease: living well. Lancet Respir Med 2017; 5: 968-980.

36 Harding R, Murtagh FE. Palliative care for management of small-cell lung cancer. Lancet 2006; 367: 474.

37 Bajwah S, Ross JR, Wells AU, et al. Palliative care for patients with advanced fibrotic lung disease: a randomised controlled phase II and feasibility trial of a community case conference intervention. Thorax 2015; 70: 830-839.

38 Bajwah S, Higginson IJ, Ross JR, et al. The palliative care needs for fibrotic interstitial lung disease: a qualitative study of patients, informal caregivers and health professionals. Palliat Med 2013; 27: 869-876.

39 Higginson IJ, Bausewein C, Reilly CC, et al. An integrated palliative and respiratory care service for patients with advanced disease and refractory breathlessness: a randomised controlled trial. Lancet Respir Med 2014; 2: 979-987. 